\title{
Helical Pitch Parameter Dependency of High Beta Equilibrium of Helical Plasmas
}

\author{
Tsuguhiro WATANABE and Hitoshi HOJO ${ }^{1)}$ \\ National Institute for Fusion Science,322-6 Oroshi-cho, Toki 509-5292, Japan \\ 1) Plasma Research Center, University of Tsukuba, Tsukuba 305-8577, Japan
}

(Received 7 December 2009 / Accepted 25 March 2010)

\begin{abstract}
The helical pitch parameter $(\gamma)$ dependency of equilibrium and the stability of the high beta plasma in the LHD type magnetic configuration is studied numerically. It is confirmed that the small $\gamma$ configurations are favorable for the LHD-type fusion reactors in the point of robustness of high beta equilibrium, compatibility of easy ignition and high output power of core plasma, in addition to a sufficient space for blankets.
\end{abstract}

(c) 2010 The Japan Society of Plasma Science and Nuclear Fusion Research

Keywords: LHD, high beta, stability, equilibrium

DOI: $10.1585 /$ pfr.5.S2022

\section{Introduction}

LHD type magnetic configuration is produced by continuous helical coil systems. The helical pitch parameter $\gamma \equiv a_{\mathrm{c}} \cdot k$ characterizes the magnetic configuration of helical systems, where $a_{\mathrm{c}}$ and $k$ are the current center radius and the wave number of the helical coils, respectively.

The LHD experimental results of achieving average beta value 5\% without the beta collapse suggests the possibility of the helical equilibriums with ultrahigh beta MHD stable core plasmas. The existence of the MHD stable high beta core plasma lead the way for the realization of economic fusion power systems.

LHD-type reactors require a large major radius $\left(R_{\mathrm{c}}\right)$ to attain the self-ignition condition with a sufficient space for blankets. To reduce the major radius size, small $\gamma$ configuration is considered [1]. On the other hand, small $\gamma$ configuration requires relatively high current density $\left(J^{c}\right)$ for helical coils and the volume of the last closed magnetic flux surface $\left(V_{\text {lcfs }}\right)$ of vacuum field become small (Fig. 1).

The helical pitch parameter dependency of equilibrium and the stability of the high beta plasma is studied numerically. For this purpose, we have developed a new numerical scheme based on Biot-Savart law. For simplicity, we describe the method for the straight helical systems (Fig. 2), from here.

\section{Numerical Method to Solve Helical Equilibrium}

MHD equations $\boldsymbol{\nabla} P=\boldsymbol{J} \times \boldsymbol{B}$ are possible to be solved without approximations, under the rotating helical coordinate system $(X, Y, \zeta)$ [2], which rotates in synchronization with helical coils. $\zeta$ is the axial coordinate. Arbitrary functions $P(\Psi)$ and $I(\Psi)$ are introduced and plasma current is

author'se-mail: wata@toki-fs.jp

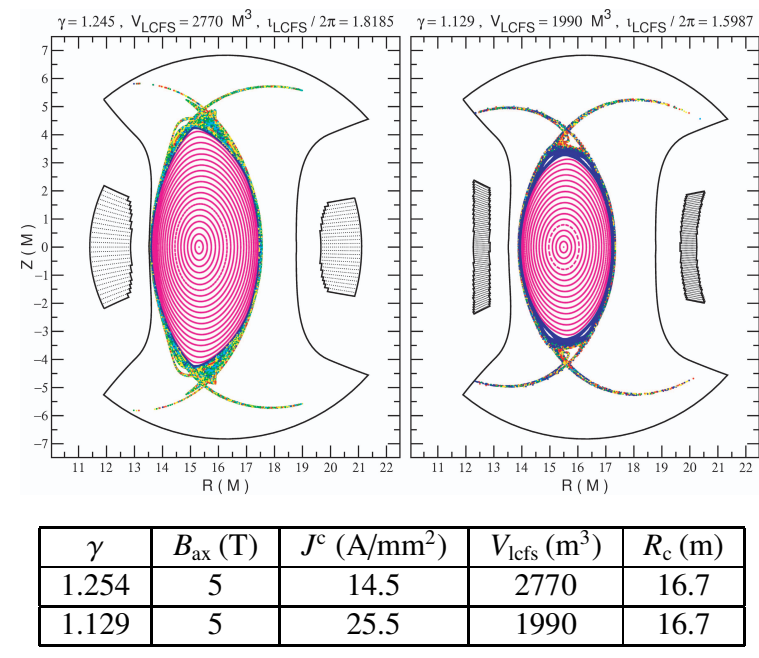

Fig. 1 Vacuum magnetic surface and blanket space of standard LHD $\gamma$ (left) and small $\gamma$ (right) configuration.

expressed as follows,

$$
\boldsymbol{J}=\frac{1}{\mu_{0}} I^{\prime}(\Psi) \boldsymbol{B}+P^{\prime}(\Psi)\left(\begin{array}{r}
-k Y \\
k X \\
1
\end{array}\right)
$$

where $k$ is the axial wavenumber of helical coils, $\Psi$ is the flux function and $P(\Psi)$ is the plasma pressure distribution. The first term of the right-hand side of eq. (1) is the driven current term, which is independent to the plasma pressure. $X$ and $Y$ components of the second term of eq. (1) are the diamagnetic currents, which produce magnetic field on the inside of the plasma column, mainly. The $\zeta$ components of the second term of eq. (1) is the bootstrap currents, which produce magnetic field on the outside of the plasma column, mainly. Magnetic field $\boldsymbol{B}$, vector potential $\boldsymbol{A}$ and the magnetic flux function $\Psi$ can be calculated by Biot-Savart 


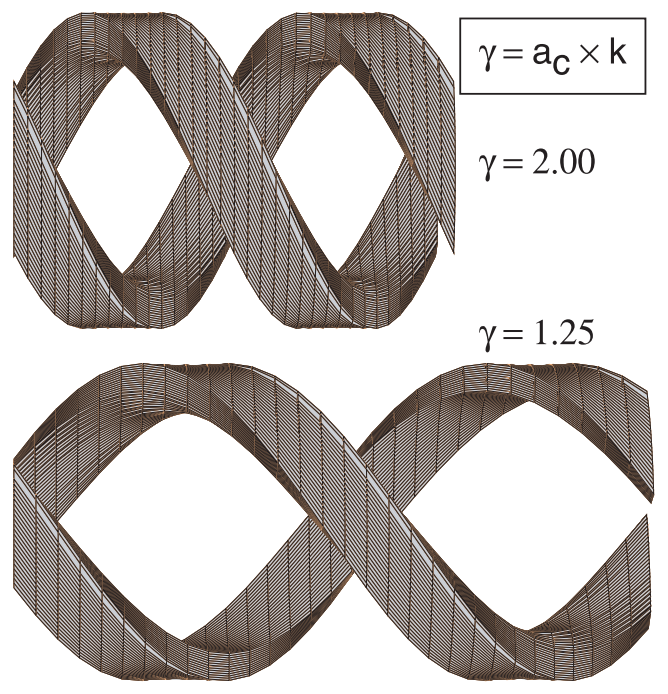

Fig. 2 Straight helical coils.

law as follows,

$$
\begin{aligned}
& \boldsymbol{B}(\boldsymbol{r})=\frac{\mu_{0}}{4 \pi} \int \mathrm{d}^{3} \boldsymbol{r}^{\prime}\left\{\frac{\left(\boldsymbol{r}^{\prime}-\boldsymbol{r}\right) \times \boldsymbol{J}^{\mathrm{s}}\left(\boldsymbol{r}^{\prime}\right)}{\left|\boldsymbol{r}^{\prime}-\boldsymbol{r}\right|^{3}}\right\}, \\
& \boldsymbol{A}(\boldsymbol{r})=\frac{\mu_{0}}{4 \pi} \int \mathrm{d}^{3} \boldsymbol{r}^{\prime}\left\{\frac{\boldsymbol{J}^{\mathrm{s}}\left(\boldsymbol{r}^{\prime}\right)}{\left|\boldsymbol{r}^{\prime}-\boldsymbol{r}\right|}-\frac{\boldsymbol{J}^{\mathrm{s}}\left(\boldsymbol{r}^{\prime}\right)}{\left|\boldsymbol{r}^{\prime}\right|}\right\}, \\
& \Psi(\boldsymbol{r})=A_{\zeta}+k\left(X A_{Y}-Y A_{X}\right), \\
& \boldsymbol{J}^{\mathrm{s}}\left(\boldsymbol{r}^{\prime}\right) \equiv \boldsymbol{J}\left(\boldsymbol{r}^{\prime}\right)+\boldsymbol{J}^{\mathrm{c}}\left(\boldsymbol{r}^{\prime}\right) .
\end{aligned}
$$

Plasma equilibrium is reduced to the following relation,

$$
\Psi(\boldsymbol{r})=\Psi^{\mathrm{p}}(\boldsymbol{r})+\Psi^{\mathrm{c}}(\boldsymbol{r}),
$$

where $\Psi^{\mathrm{p}}(\boldsymbol{r})$ is the flux function produced by the plasma current whose profile is determined by the "total" flux function $\Psi(\boldsymbol{r})$ through the eq. (1). $\Psi^{\mathrm{c}}(\boldsymbol{r})$ is the flux function produced by the helical coil currents. Plasma equilibrium computations are reduced to solve eq. (6) self-consistently for $\Psi$. The relaxation scheme is possible to solve eq. (6). In the following we have assumed that the driven current is zero $\left(I^{\prime}(\Psi)=0\right)$ and pressure profile is one of a flat top type, using the value of the flux function at separatrix, $\Psi_{\mathrm{s}}$.

$$
P(\Psi)=\beta_{\mathrm{ax}} \frac{\boldsymbol{B}_{\mathrm{ax}}^{2}}{2 \mu_{0}} \exp \left\{-D\left(\frac{\Psi}{\Psi_{\mathrm{s}}}\right)^{2}\right\}, \quad D=7
$$

\section{MHD Stability and its Helical Pitch Parameter Dependency}

Plasma stability is determined by the MHD potential energy [3],

$$
W=\int \mathrm{d} V\left(\frac{3}{2} P+\frac{1}{2 \mu_{0}} \boldsymbol{B}^{2}\right) \equiv W_{\mathrm{T}}+W_{\mathrm{B}} .
$$

$W$ minimum configuration is an MHD stable equilibrium. When $\delta W<0\left(\delta W=W-W_{0}, W_{0} \equiv \int \mathrm{d} V \frac{1}{2 \mu_{0}} \boldsymbol{B}_{\text {ext }}{ }^{2}\right)$, transition to vacuum state is energetically prohibited. Beta collapse of core plasma does not occur.

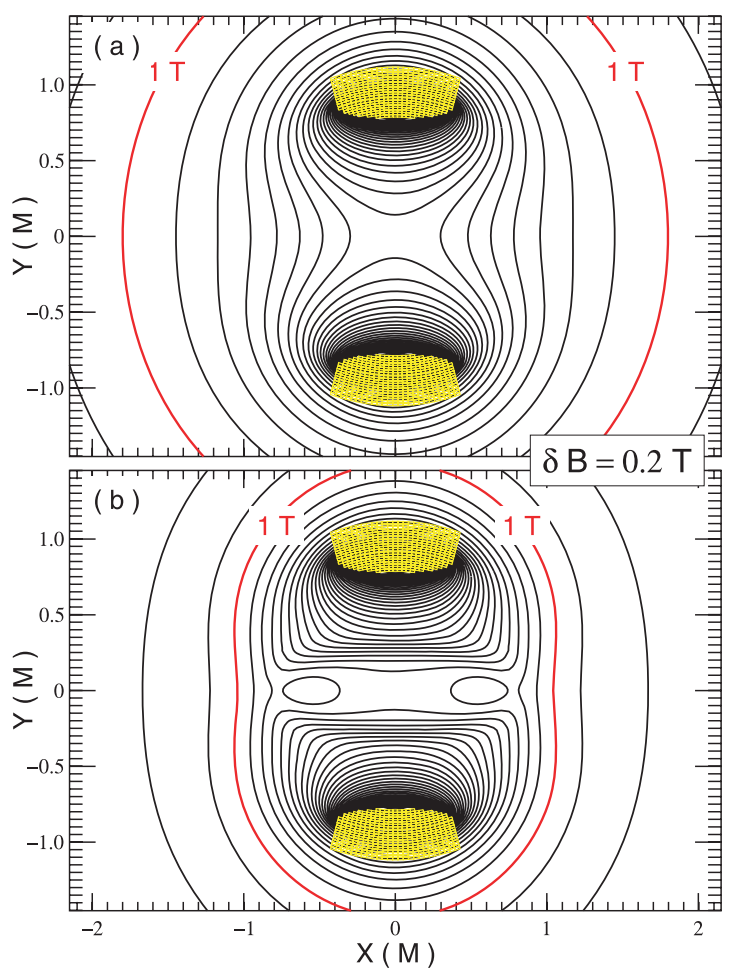

Fig. 3 Field intensity, $|\boldsymbol{B}|$, distributions of vacuum and high beta state $\left(\beta_{\mathrm{ax}}=176 \%\right)$ are shown in (a) and (b). $\gamma=1.1221$.

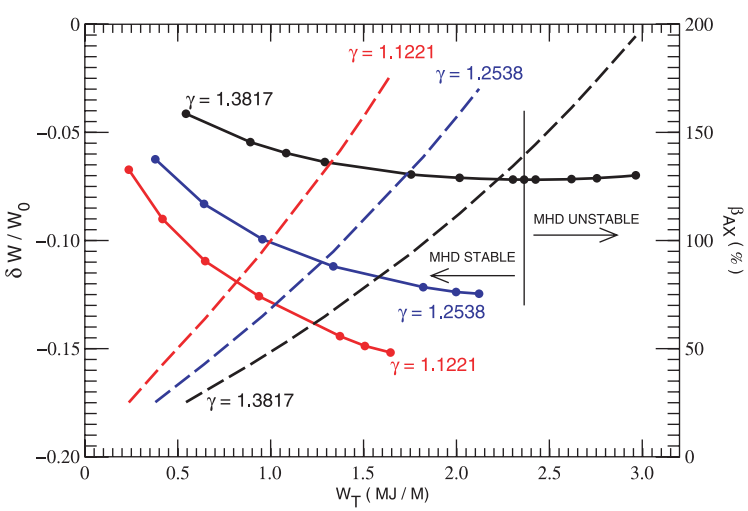

Fig. 4 The solid lines show relations between variations of the MHD potential energy $\delta W$ and thermal energy $W_{\mathrm{T}}$ stored in the magnetic surface. Broken lines show the beta values $\beta_{\mathrm{ax}}$ at the magnetic axis.

When the bootstrap current cancels, partially, the magnetic field outside the helical coils, the magnetic field energy $W_{\mathrm{B}}$ can be reduced extensively, as shown in Fig. 3. The variation of potential energy become negative $(\delta W<$ 0 ) by plasma sustainment and bootstrap transition to high beta equilibrium occur.

Helical pitch parameter dependency of high beta equilibrium is summarized in Fig. 4.

When $\gamma$ is small, the size of the magnetic surface become small, since the axial magnetic field decreased relatively. However, the ability of MHD stability increases since the role of bootstrap current is increased. This ten- 


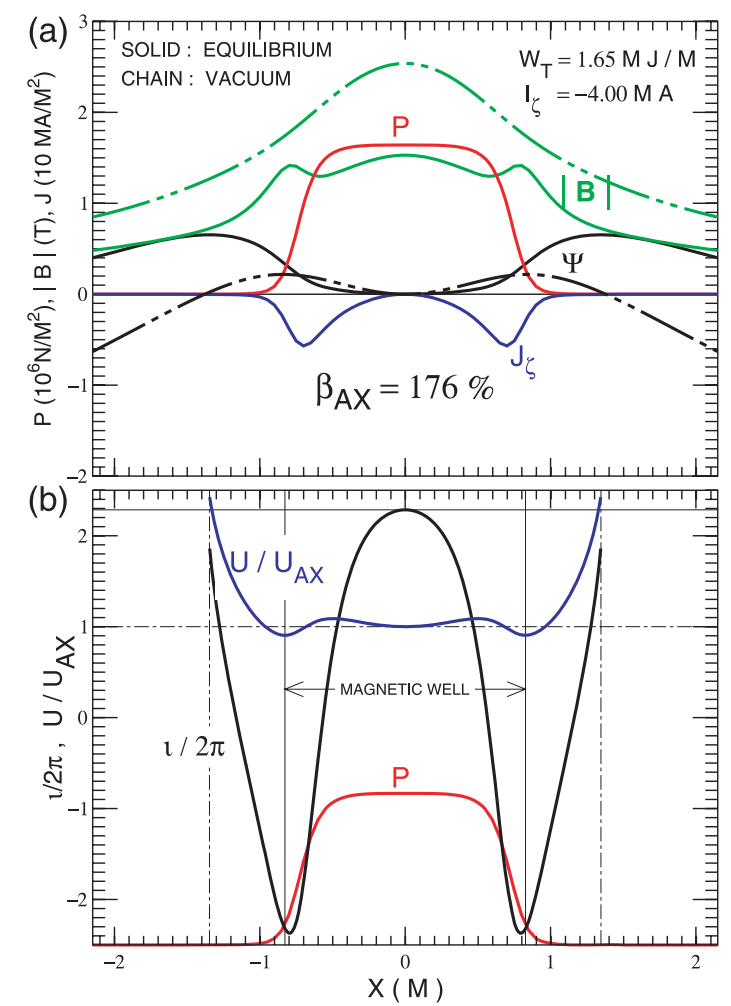

Fig. 5 (a) Pressure, bootstrap current, flux function and magnetic field intensity along $X$ coordinate. (b) Specific volume $U$ and rotational transform $\iota / 2 \pi$. Pressure profile is superimposed in this graph.

dency is shown by the plot of MHD potential energy $\delta W$ for the case of $\gamma=1.1221$ in Fig. 4.

The value of the MHD potential energy, $W$, depend on the functional form of equilibrium pressure distribution $P(\Psi)$. The core plasma has a possibility of transition to a equilibrium distribution function $P(\Psi)$, which minimize the potential energy $W$, at the specified value of the stored thermal energy $W_{\mathrm{T}}$. Computations of the MHD potential energy, $W$, has predicted that peaked pressure profile,

$$
P(\Psi)=\beta_{\mathrm{ax}} \frac{\boldsymbol{B}_{\mathrm{ax}}^{2}}{2 \mu_{0}} \exp \left\{-D\left(\frac{\Psi}{\Psi_{\mathrm{s}}}\right)\right\}, \quad D=7,
$$

has a possibility of collapsing to the flat top type pressure profile given by eq. (7). However, the ultrahigh beta equilibrium with the flat top type pressure profile given by eq. (7) will be almost minimize the MHD potential energy $W$, because local linear stability criterion [4] is satisfied by the combination of the high magnetic shear at peripheral region and the magnetic well at inner region of the magnetic surface, as shown in Fig. 5.

Figure 5 shows profiles of equilibrium quantities along the long axis of magnetic surface for the case of $\beta_{\mathrm{ax}}=176 \%$. The magnetic surface volume $V_{\text {lcfs }}$ grows by 2.4 times compared with the magnetic surface volume at the vacuum state.

\section{Summary}

Summary is as follows.

- Small helical pitch parameter configuration has a lot of advantages for the LHD-type fusion reactors.

- The heating systems for the ignition become small because the vacuum magnetic surface volume is small.

- After the ignition, fusion output power is large due to the large volume of the high beta core plasma.

- Core plasma is robust against MHD perturbations.

- The ultrahigh beta equilibrium has enough intensity in the magnetic field for the alpha-particle confinement.

- The space for the blanket is large.

This work is performed with the support and under the auspices of the NIFS Collaborative Research Program NIFS08KDBD006, NIFS09KTBL010 and NIFS08KNXN144.

[1] S. Imagawa, A. Sagara and Y. Kozaki, Plasma Fusion Res. 3, S1050 (2008).

[2] T. Watanabe et al., Nucl. Fusion 46, 291 (2006).

[3] G. Bateman, MHD Instabilities (MIT Press, Cambridge, Massachusetts, and London, England, 1980) p.38.

[4] T. Watanabe and H. Hojo, J. Plasma Fusion Res. Ser. 5, 487 (2003). 